\title{
Radio Frequency Tank Circuit for Probing Planar Lipid Bilayers
}

\author{
Abhishek Bhat ${ }^{1}$, Jonathan Rodriguez ${ }^{1}$, Hua Qin ${ }^{2}$, Hyun Cheol Shin ${ }^{1}$, Hyuncheol Shin ${ }^{1}$, Joerg Clobes ${ }^{1}$, \\ Dustin Kreft ${ }^{1}$, Jonghoo Park ${ }^{3}$, Eric Stava ${ }^{1}$, Minrui Yu ${ }^{1}$, Robert H. Blick ${ }^{1,4}$ \\ ${ }^{1}$ Laboratory for Molecular-Scale Engineering, Department of Electrical and Computer Engineering, University of Wisconsin-Madi- \\ son, Madison, USA; ${ }^{2}$ Laboratory of Nanodevices and Application, Suzhou Institute of Nanotech and Nanobionics, Chinese Academy \\ of Sciences, Beijing, China; ${ }^{3}$ Electrical Engineering, Kyungpook National University, Daegu, Republic of Korea; ${ }^{4}$ Genome Center of \\ Wisconsin, University of Wisconsin-Madison, Madison, USA. \\ Email: abhat@wisc.edu
}

Received July $27^{\text {th }}, 2013$; revised August $27^{\text {th }}, 2013$; accepted September $5^{\text {th }}, 2013$

Copyright (C) 2013 Abhishek Bhat et al. This is an open access article distributed under the Creative Commons Attribution License, which permits unrestricted use, distribution, and reproduction in any medium, provided the original work is properly cited.

\begin{abstract}
We present first results from a hybrid coplanar waveguide microfluidic tank circuit for monitoring lipid bilayer formation and fluctuations of integrated proteins. The coplanar waveguide is a radio frequency resonator operating at $\sim 250$ $\mathrm{MHz}$. Changes within the integrated microfluidic chamber, such as vesicle bursting and subsequent nanopore formation alter the reflected signal, and can be detected with nanosecond resolution. We show experimental evidence of such alterations when the microfluidic channel is filled with giant unilamellar vesicles (GUVs). Subsequent settling and bursting of the GUVs form planar lipid bilayers, yielding a detectable change in the resonant frequency of the device. Results from finite element simulations of our device correlate well with our experimental evidence. These simulations also indicate that nanopore formation within the bilayer is easily detectable. The simulated structure allows for incorporation of microfluidics as well as simultaneous RF and DC recordings. The technique holds promise for high throughput drug screening applications and could also be used as an in-plane probe for various other applications. It opens up possibilities of exploring ion channels and other nano scale pores in a whole new frequency band allowing for operating at bandwidths well above the traditional DC methods.
\end{abstract}

Keywords: Radio Frequency; Simulations; Lipid Bilayers; Ion Channels; High Speed; Planar Detection

\section{Introduction}

Information processing in biological systems is a highly complex and parallel task. Cells rely on the conduction of ions through the cell membrane via membrane embedded ion channel proteins for intercellular communication. The investigation of these channels in cell membranes as well as artificial planar lipid bilayers is of paramount importance for pharmaceutical applications. Electrical measurements of ion channels are traditionally carried out in the DC regime using the well-established patch clamp technique [1] and, more recently, the on-chip planar patch clamping technique [2]. However Ramachandran et al. [3,4], have shown that the dwell time of ions in the channel, which is on the order of nanoseconds, can be probed with radio frequency (RF) devices. While dielectric spectroscopy of biological molecules has been reported $[5,6]$, the use of RF signals for direct investigation of ion channels has not been studied in great detail. In order to yield real-time, in vitro information of ion channel operation, it is essential to have circuitry available that can deliver RF spectroscopy of ion transport. We accomplish this with an easy to use on-chip method, which is essential for the adoption of this technique. Here, we present first data from this technique for the detection of planar lipid bilayer formation, a crucial first step for the RF monitoring of ion channels.

A challenge in the design of RF circuitry for probing cellular signals is the high attenuation presented by the aqueous solution containing cells or lipids [7]. We overcome this problem by integrating microfluidics with a coplanar waveguide (CPW). This hybrid device (see Figure 1(a)) confines a very small and localized amount of fluid volume to the sensing surface and probes with nanosecond resolution. Thus the screening effect of the liquid is substantially reduced. The reflected signal from our RF "tank" circuit then acts as a probe for the specimen in the microfluidic 
(a)

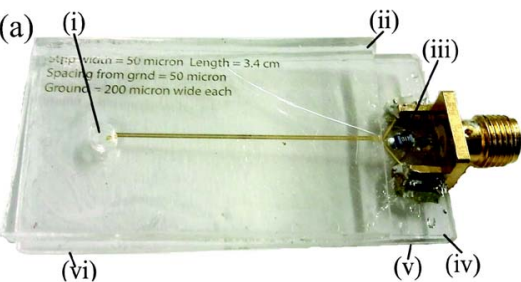

(c)

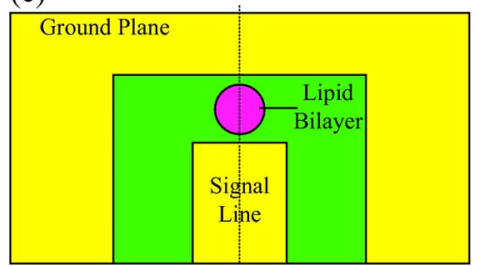

(b)

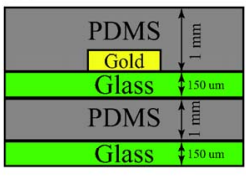

(d)

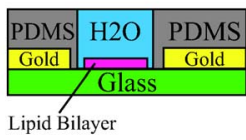

Figure 1. (a) Fabricated device: (i) pore for fluids, (ii) top PDMS layer, (iii) inductor, (iv) top glass substrate, (v) botom glass, (vi) bottom PDMS layer; (b) device layer squence; (c) waveguide sensing region: dotted line indicates the line of cut for section (d); (d) sidecut of the sensing region (not to scale).

device. This setup provides increased sensitivity and a fast readout capability, as evidenced by Schoelkopf et al. [8,9] for single electron transport. A similar planar device was presented by Wood et al. for coulter counting applications [10] at the micro scale. Utilizing a narrow range of high frequencies reduces noise and improves the signal-to-noise ratio, allowing us to perform high speed time domain measurements with nanosecond resolution. Details on the operation of the tank circuit are provided below.

\section{Fabrication}

The device is shown in Figure 1(a): the primary sensing element is a CPW structure formed by gold deposition on a $150 \mu \mathrm{m}$ thick glass substrate (microscope cover slip, Fisher Sci., Cat. No. 12-545-88). Standard lithography processes are followed for device fabrication. Before metal deposition, the samples were subjected to an oxygen plasma in order to enhance adhesion between metal and the glass. A $150 \mathrm{~nm}$ Au layer was deposited on a 5 $\mathrm{nm}$ Ti adhesion layer to define the CPW. We sandwich a thin sheet of Polydimethylsiloxane (PDMS) (Dow Corning, Sylgard 184 Silicone Elastomer) between the device glass and a base glass cover slip. An additional PDMS sheet with a pre-drilled pore was added on top of the coplanar waveguide structure. The pore could also be used in simultaneous DC measurements using a planar patch clamp setup. A technique presented by $\mathrm{Yu}$ et al. [11], is ideal for drilling smooth pores in the glass substrate to carry out simultaneous RF/DC planar patch clamp measurements. All layers were bonded using uncured PDMS for strong adhesion. Silver conductive epoxy was then applied to glue a surface mount inductor and an SMA connector to the waveguide input.

As shown in Figure 1(c), the end of the signal line is left open so that it acts as a capacitor, as suggested by Beilenhoff et al. $[12,13]$ The signal line of the CPW is 50 $\mu \mathrm{m}$ wide and $3.4 \mathrm{~cm}$ in length. The ground planes on either side of the signal line are $200 \mu \mathrm{m}$ wide with a spacing of $50 \mu \mathrm{m}$ between the ground planes and signal line. The gap between the signal line and the ground plane at the open circuited end is $150 \mu \mathrm{m}$.

\section{Simulation Setup}

Simulations were carried out with Ansoft HFSS [14]. For simulation purposes, we assume the PDMS sheets to have a thickness of $1 \mathrm{~mm}$ and define the relative permittivity to be 2.5 [15]. The pore in the top PDMS layer is aligned with the end of the CPW structure and is designed for the addition of microliters of fluids. A representation of the different layers used in the device is shown in Figure 1(b).

Figure 1(d) represents a sidecut taken through the centre of the signal line, showing the ground plane and the signal line on either side of the lipid bilayer. The lipid bilayer was modelled as a sheet with a $4 \mathrm{~nm}$ thinness and a relative permittivity of $\varepsilon_{r}=2$. The inductor value selected is $100 \mathrm{nH}$. In our simulations, we define the sensing region of the device using a cuboid with a $750 \mu \mathrm{m}^{2}$ base and $50 \mu \mathrm{m}$ height. The cuboid is located in the gap between the end of the signal line and the ground plane. The gradient of the electric field is highest in this region (discussed later) and hence makes the device sensitive to any perturbations in this region. Figure 1(c) shows this sensing region, where the position of the lipid bilayer is indicated in pink.

\subsection{Circuit Behaviour}

The inset in Figure 2(a) shows a simple circuit equivalent of our setup. The two energy storage elements, the capacitor and the inductor, together form what is commonly referred to as a tank circuit. The impedance offered by the inductor is $\mathrm{j} \omega \mathrm{L}$ while that offered by the capacitance is $1 / j \omega \mathrm{C}$ where $\mathrm{L}$ and $\mathrm{C}$ are the inductance and capacitance values respectively. This setup causes the circuit to resonate at a frequency approximated by $\mathrm{f}=$ $1 / 2 \pi \sqrt{ }(\mathrm{LC})[16]$. At this frequency most of the power sent into the circuit is absorbed and very little is reflected back to the input port. Variations in the system's dielectric permittivity due to the formation of the lipid bilayer lead to an alteration in the circuit capacitance, which is detected as a change in the resonant frequency.

The reflectance curve indicates the power loss of the reflected signal in comparison to the input signal power. The frequency of the resonance is defined by the circuit inductance and capacitance. The width of the resonance curve and the signal strength are dictated by the resis 
(a)
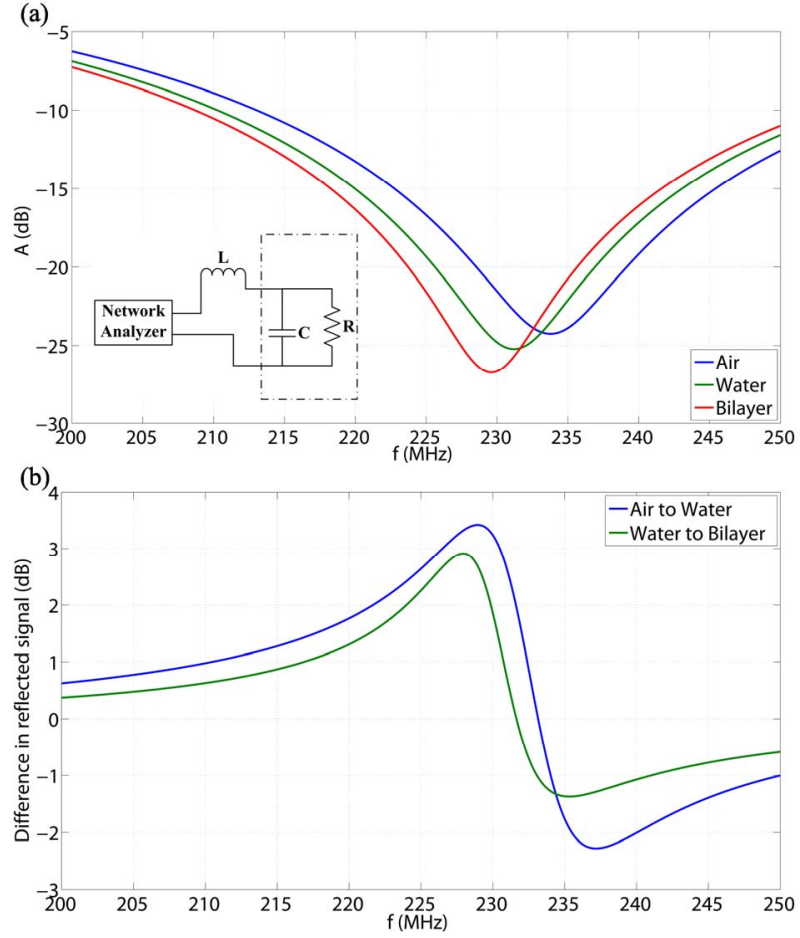

Figure 2. (a) Simulated reflectance of the circuit for different conditions. The inset shows the equivalent circuit diagram of the setup, the dotted region indicates the circuit equivalent of the coplanar waveguide structure along with the lipid bilayer; (b) Indicates the difference in amplitude going from air to water and from water to lipid bilayer formation.

tance and other losses associated with the non ideal nature of $\mathrm{L}$ and $\mathrm{C}$. The quality of the peak is described by the quality (Q) factor, a measure of the "sharpness" of the resonant peak. It can be defined as the ratio of the resonant frequency to the bandwidth at the half power frequencies. In general a higher $\mathrm{Q}$ factor indicates a better resonator and a sharper peak.

\section{Simulation Results}

Figure 2(a) shows results from the reflectance (S11) simulations for devices with air, water and a lipid bilayer in the sensing region. Our simulations reveal a detectable change in the reflectance with the incorporation of deionized (DI) water and a further significant change with the formation of a lipid bilayer. When the microfluidic chamber is filled with air, there exists a well-defined resonance peak in the reflectance signal at $234 \mathrm{MHz}$. This resonance shifts down in frequency by $2.7 \mathrm{MHz}$ when DI water is introduced and again by $1.6 \mathrm{MHz}$ when a bilayer forms in the sensing region. The amplitude of the resonant peak changes by 0.98 and $1.5 \mathrm{~dB}$ for each frequency shift respectively.

Figure 2(b) highlights the change in amplitude when water is introduced into the system and, subsequently, when a lipid bilayer forms in the sensing region. Peaks in this trace represent the frequencies at which the reflectance measurements are most sensitive to changes in the sensing region. The sensitivity is highest on the shoulder of the resonant peak at $228 \mathrm{MHz}$. This is the ideal point of operation for time domain measurements. There is a $3.3 \mathrm{~dB}$ change in response with the introduction of water and a $2.9 \mathrm{~dB}$ change when the bilayer forms. These shifts in amplitudes correspond to voltage variations that are easily detectable in a time domain measurement setup. In general the temporal resolution is given by $1 / \mathrm{f}$ and a high operating frequency is desired for the device.

\subsection{Electric Field Distribution}

Figure 3 shows the electric field distribution around the centre of the sensing region, with a cut taken along the length of the device (Figure 1(c)) in the absence (Figure 3(a)) and presence (Figure 3(b)) of a lipid bilayer. Figure 3(a) demonstrates that even a small volume of water

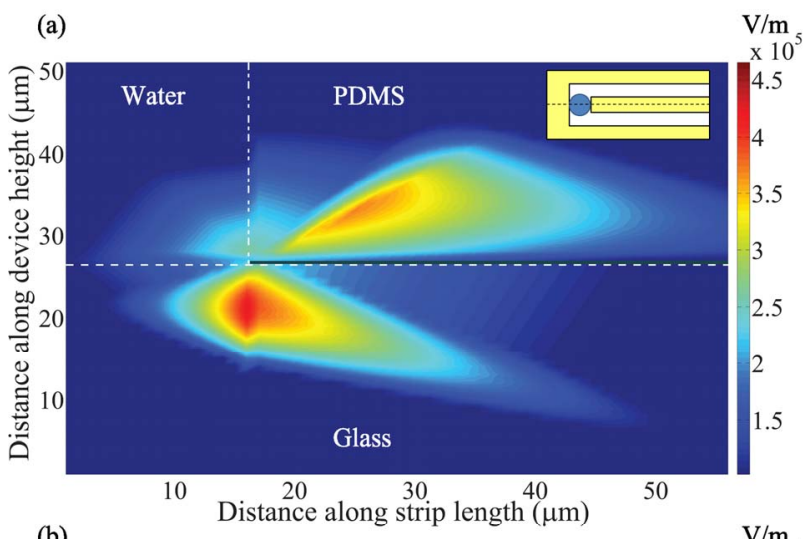

(b)

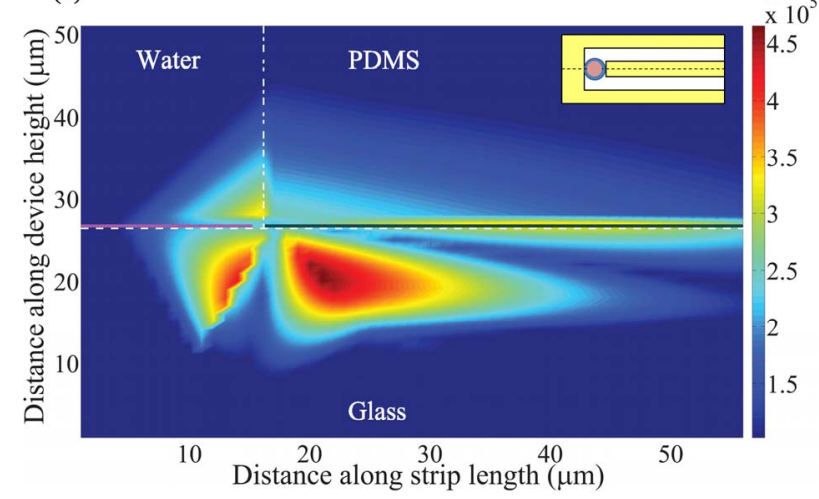

Figure 3. Electric field distribution around the centre of the sensing region, with a cut taken along the length of the device (Figure 1(c)) in the absence (a) and presence (b) of a lipid bilayer. Dotted white lines represent the interface between different device layers. The signal line is shown in dark green at the PDMS-glass interface while the lipid bilayer is seen in pink at the water-glass interface. $x$ and $y$ axes denote length in microns. Insets show a schematic of the device in each case with the dotted line showing plane of cut. 
has a substantial screening effect on the electric field. This, however, still permits for an electric field of significant magnitude at the centre of the sensing region, as evidenced in both Figures 3(a) and (b). The field in the PDMS structure deteriorates with bilayer formation while the field over the bilayer increases, demonstrating that the presence of the bilayer influences the electric field to a large extent. This is expected since the permitivity of the bilayer differs greatly from that of water. This region of strong electric field over the lipid bilayer forms the sensing region of the device, as defined in Figure 1.

\section{Experimental Results}

Figures 4(a) and (b) show experimental results from the fabricated device (shown in Figure 1) when different solutions were added to the pore in the PDMS top layer. To study the response of the system, we used DI water and giant unilamellar vesicles (GUVs) suspended in DI water. The GUVs, consisting of the DPhPC molecule, were formed in DI water. Allowing the vesicle suspension to diffuse to the glass substrate enables GUV bursting on the substrate and subsequent bilayer formation. Controlled experiments were carried out with a 20 minute interval between each experiment and the responses were very repeatable. The fluid chamber was flushed with DI water and isopropyl alcohol between experiments, allowing the microfluidic chamber to dry prior to each new experiment.

GUV bursting was used due to the confined operating space that the microfluidic chamber presents and a pre-existing setup to make the vesicles. The technique has several other advantages with the given setup. When compared to the adsorption and bursting of small or large
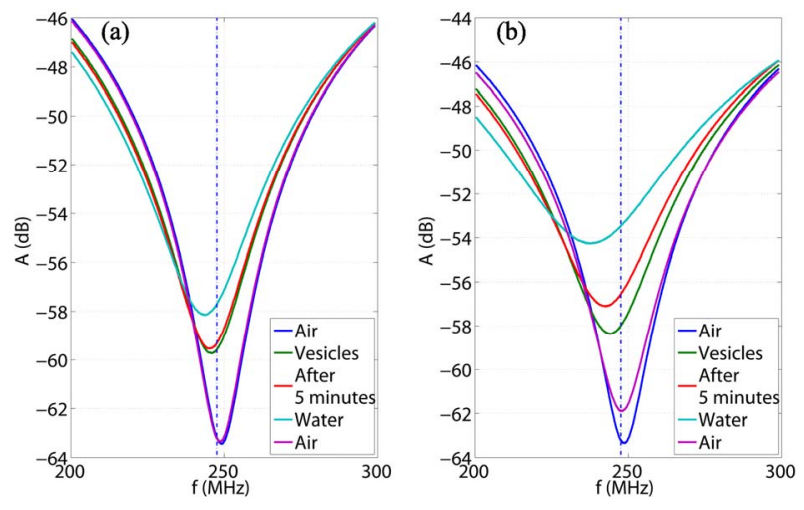

Figure 4. The measured reflected signal under different conditions. The device response was measured for air, after introduction of vesicles suspended in DI water, after allowing the vesicles to settle for 5 minutes, replacing the suspension with only DI water and after removal of the DI water. Dotted line indicates the best frequency of operation for the device. (a) First set of measurements and (b) second set of measurements. unilamellar vesicles, GUV bursting has the advantage of being able to cover a larger area. Furthermore, because making the sensing region clean and hydrophilic is difficult due to the proximity of the gold patterning, the probability of obtaining a large lipid bilayer within the sensing region is small when other techniques are used.

Figure 4 makes clear that there is a substantial increase in the reflected signal strength when vesicles are added to the system. More importantly, there is an even greater increase in the reflected signal when the vesicles are allowed to settle in the sensing region, which we attribute to lipid bilayer formation. The changes that may occur due to multiple layers or vesicle adsorption will need simultaneous DC investigation which shall be carried out at a later stage. We see another substantial jump in signal strength when the vesicle solution is replaced by DI water. Because the vast majority of the vesicle solution's composition is DI water, this result demonstrates that the device is sensitive enough to detect even the smallest of differences in permittivity.

We observe that there is a decrease in the resonant frequency of up to $10 \mathrm{MHz}$ when DI water is added to the system, and a $5 \mathrm{MHz}$ shift in response after adding the vesicle solution. These are both substantial shifts that are easily detectable in the time and frequency domains. A smaller, but still detectable shift is seen after the vesicles are allowed to settle on the device. We achieve this by leaving the setup undisturbed for 5 minutes after the vesicle solution is introduced into the pore. When the device is flushed and dried, the response returns to its original shape.

There is good agreement between the simulation results of Figure 2(a) and the measured data. While the simulation suggests a $\mathrm{Q}$ factor of 26 , the fabricated device has a $Q$ factor of 19 . The predicted change in amplitude upon addition of water to the system is $3.42 \mathrm{~dB}$ while the device itself shows a change of 6 to $8 \mathrm{~dB}$. In the case of lipid bilayer formation, the simulation predicts a change of $2.91 \mathrm{~dB}$ while the actual results show a change of $1.6-3.15 \mathrm{~dB}$. Also, as suggested by the simulation, operating on the shoulder of the resonance curves at $248 \mathrm{MHz}$ gives the best detection capability.

\section{Simulations for Future Work}

To simulate ion channels and other nanopores, we introduced a $5 \mu \mathrm{m}$ pore in the substrate glass slide (Figures $\mathbf{1 ( a - i v ) ) . ~ T h i s ~ p o r e ~ i s ~ l o c a t e d ~ i n ~ t h e ~ c e n t e r ~ o f ~ t h e ~ s e n s i n g ~}$ region. The bottom PDMS (Figures 1(a-vi)) was replaced by saline water. Saline was also assumed to occupy the pore in the substrate and the top PDMS layer (Figures 1(a-i)). When representing the ion channel, we introduced a $4 \mathrm{~nm}$ pore in the lipid bilayer. Such a pore could also represent any other nanopore formed by a pore-forming protein, such as alpha hemolysin [3]. Figure 5 shows the results of this simulation. 


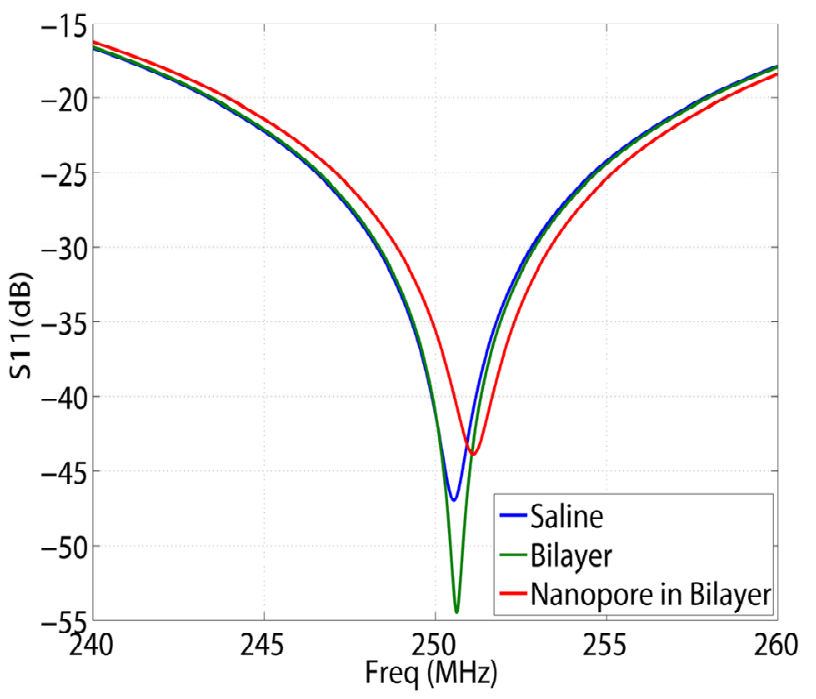

Figure 5. Simulated reflectance measurement for detection of an ion channel in the lipid bilayer system. The traces show the predicted reflectance upon formation of a lipid bilayer on the device and upon formation of a nanopore in this bilayer.

The simulation predicts a change in the reflected signal amplitude along with a slight shift of resonant frequency when a lipid bilayer forms on the substrate. There is another shift in the resonant frequency and a $10 \mathrm{~dB}$ change in amplitude when a nanopore is introduced into the lipid bilayer. These changes are well within the detection capabilities of most high frequency equipment and will allow for the monitoring of subtle events such as molecules passing through the nanopore.

\section{Discussion and Future Work}

Due to the presence of the bottom PDMS layer, the currently fabricated device is incompatible with the drilling of the through hole and the incorporation of fluids under the substrate. These processes, however can easily be done in future with some minor modifications to the device fabrication and setup. Experiments with this new setup shall allow us to measure the DC capacitance of the system for verification of a stable bilayer formation. Additionally, they will allow for simultaneous RF and DC nanopore measurements. While our simulations show a change of about $60 \mathrm{fF}$ in the capacitance of the device-a value calculated based on the resonant frequency, assuming the LC circuit model shown in the inset of Figures 2(a) upon formation of the bilayer, this number just predicts the capacitance change seen by the RF line for the current orientation. A traditional measurement of the bilayer capacitance as commonly reported in literature can only be performed when the electric field is perpendicular to the bilayer, which would be the case in a DC measurement.
By controlling the pore size, the inductor value and the waveguide geometry, it is possible to adjust the resonant peak over a wide frequency range, thus fine-tuning the sensitivity. This suggests that nanosecond time resolution readouts of cellular events can potentially be obtained by carrying out time domain reflectance measurements using a simple directional coupler. Our device measurements validate these simulation results to a large extent.

The variations in the simulated and measured data can be attributed to the imprecise alignment of the PDMS pore over the coplanar waveguide. Also, the simulation assumes only a single lipid bilayer centred in the middle of the sensing region, while there are likely multiple such layers on the device or a conglomeration of lipids in the sensing region. Liquid leakage into the small gap between the top PDMS and the glass may also cause the variations. We are in the process of developing a better fabrication method in order to reduce these issues. Most of these factors can be compensated for by conducting a simultaneous DC recording, as mentioned previously, in order to better characterize the device.

\section{Conclusion}

We have shown that a tank circuit for bilipid data recordings can be realized and yield information on membrane formation. We detected lipids in the sensing region and found that the device behaviour is in close agreement with our simulation results. Based on these simulations and early experimental findings, our system has the potential for carrying out simultaneous RF and DC measurements of ion channels. The technique has applications in a variety of areas. While it holds promise for high sampling rate drug screening applications, it also opens up possibilities for exploring ion channels and other pore forming proteins in the radio frequency band. Our low-cost device could prove to be a useful tool for a variety of pharmaceutical and research applications. Other applications range from studying real time transitions of ions through pores to whole cell or cell attached planar patch clamp measurements in the RF domain. One particularly simple example of applying the setup is an RF, high speed Coulter counter for use in various fields. Additionally, the planar detection scheme employed in our device can be used in a host of other applications. Because the extremely thin form factor allows the sensing element to be patterned into soft and flexible materials, the device can be used in conjunction with existing methods along with incorporated microfluidics and auxiliary electrical and optical measurements.

\section{Acknowledgements}

We would like to acknowledge support by DARPA through the MOLDICE program C.F.49620-03-1-03837. 


\section{REFERENCES}

[1] B. Sakmann and E. Neher, "Single-Channel Recording," 2nd Edition, Plenum Press, New York, 1995.

[2] N. Fertig, R. H. Blick and J. C. Behrends, "Whole Cell Patch Clamp Recording Performed on a Planar Glass Chip," Biophysical Journal, Vol. 82, No. 6, 2002, pp. 3056-3062. http://dx.doi.org/10.1016/S0006-3495(02)75646-4

[3] S. Ramachandran, D. W. van der Weide and R. Blick, "Direct Microwave Transmission on Single $\alpha$-Hemolysin Pores," Applied Physics Letters, Vol. 99, No. 9, 2011, Article ID: 093105. http://dx.doi.org/10.1063/1.3626586

[4] H. S. Kim, S. Ramachandran, E. Stava, D. W. V. D. Weide and R. H. Blick, "Radio-Frequency Response of Single Pores and Artificial Ion Channels," New Journal of Physics, Vol. 13, 2011, Article ID: 093033. http://dx.doi.org/10.1088/1367-2630/13/9/093033

[5] G. R. Facer, D. A. Notterman and L. L. Sohn, "Dielectric Spectroscopy for Bioanalysis: From $40 \mathrm{~Hz}$ to $26.5 \mathrm{GHz}$ in a Microfabricated Wave Guide," Applied Physics Letters, Vol. 78, No. 7, 2001, pp. 996-998. http://dx.doi.org/10.1063/1.1347020

[6] J. Hefti, A. Pan and A. Kumar, "Sensitive Detection Method of Dielectric Dispersions in Aqueous-Based, Surface-Bound Macromolecular Structures Using Microwave Spectroscopy," Applied Physics Letters, Vol. 75, No. 12, 1999, pp. 1802-1804. http://dx.doi.org/10.1063/1.124825

[7] S. Ramachandran, R. Blick and D. van der Weide, "Radio Frequency Rectification on Membrane Bound Pores," Nanotechnology, Vol. 21, No. 7, 2010, Article ID: 075201.

http://dx.doi.org/10.1088/0957-4484/21/7/075201

[8] R. J. Schoelkopf, P. Wahlgren, A. A. Kozhevnikov, P. Delsing and D. E. Prober, "The Radio-Frequency Single-Electron Transistor (rf-set): A Fast and Ultrasensitive Electrometer," Science, Vol. 280, No. 5367, 1998, pp. 1238-1242. http://dx.doi.org/10.1126/science.280.5367.1238

[9] H. Qin and D. A. Williams, "Radio-Frequency PointContact Electrometer," Applied Physics Letters, Vol. 88, No. 20, 2006, Article ID: 203506. http://dx.doi.org/10.1063/1.2205159

[10] D. K. Wood, M. V. Requa and A. N. Cleland, "Microfabricated High-Throughput Electronic Particle Detector," Review of Scientific Instruments, Vol. 78, No. 10, 2007, Article ID: 104301. http://dx.doi.org/10.1063/1.2794230

[11] M. Yu, H. S. Kim and R. H. Blick, "Laser Drilling of Nano-Pores in Sandwiched Thin Glass Membranes," Optics Express, Vol. 17, No. 12, 2009, pp. 10044-10049. http://dx.doi.org/10.1364/OE.17.010044

[12] K. Beilenhoff, H. Klingbeil, W. Heinrich and H. L. Hartnagel, "Open and Short Circuits in Coplanar MMIC's," IEEE Transactions on Microwave Theory and Techniques, Vol. 41, No. 9, 1993, pp. 1534-1537. http://dx.doi.org/10.1109/22.245673

[13] K. C. Gupta, "Microstrip Lines and Slotlines. The Artech House Microwave Library," 2nd Edition, Artech House, Boston, 1996.

[14] Ansoft, HFSS Version 12.0 on a PC Running Windows 7 x64 on a $2.3 \mathrm{GHz}$ dual core AMD X2 processor and $4 \mathrm{~GB}$ RAM.

[15] S. V. Patel, T. E. Mlsna, B. Fruhberger, E. Klaassen, S. Cemalovic and D. R. Baselt, "Chemicapacitive Microsensors for Volatile Organic Compound Detection," Sensors and Actuators B: Chemical, Vol. 96, No. 3, 2003, pp. 541-553. http://dx.doi.org/10.1016/S0925-4005(03)00637-3

[16] D. K. Wood, S. H. Oh, S. H. Lee, H. T. Soh and A. N. Cleland, "High-Bandwidth Radio Frequency Coulter Counter," Applied Physics Letters, Vol. 87, No. 18, 2005, Article ID: 184106. http://dx.doi.org/10.1063/1.2125111

[17] D. J. Aidley and P. R. Stanfield, "Ion Channels: Molecules in Action," Cambridge University Press, Cambridge, New York, 1996. 